\title{
The St. Petersburg Paradox and the Crash of High-Tech Stocks in 2000
}

During the late 1990s high technology growth stock prices were raised to unprecedented levels by avid stock purchasers around the world. In early 2000, share prices subsequently underwent prolonged declines, leaving many purchasers with devastating losses. This article reviews some aspects of the history of the St. Petersburg paradox and some related games. We recount a remarkable article by Durand in which the valuation of growth stocks is related to the St. Petersburg paradox. Our conclusion is that the run-up in stock prices in the late 1990s and the subsequent declines in 2000 could have been avoided by an analysis and application of the St. Petersburg paradox.

KEY WORDS: Alan Greenspan; Cleveland Indians baseball team; Fair game of chance; Geometric distribution; High technology stocks; Irrational exuberance; Mark Twain; Mutual funds; Utility function; Valuation of growth stocks.

\section{INTRODUCTION}

In the early 1700s Nikolaus Bernoulli formulated a problem in the theory of games of chance, now known as the St. Petersburg paradox. At that time, Bernoulli was a relatively new doctor of jurisprudence, having written in 1709 a dissertation at the University of Basel, Switzerland, on the application of the calculus of probability to questions arising in the practice of law. In 1731 he was appointed professor in law and he later served four times as Rector of the University of Basel. (As is well known, the Bernoullis were a renowned family of Swiss mathematicians, several members of which studied probability theory; particularly notable for his work in that field was James Bernoulli, the father of the first law of large numbers.)

On September 9, 1713, Bernoulli communicated his problem by letter to Pierre Reymond de Montmort, and this was followed by a series of letters between them. Montmort himself was perplexed by the problem, and he later published his correspondence with Bernoulli in the second edition of his book on games of chance (Montmort 1713). There, the world could read about Bernoulli's paradox for the first time, and since then the problem has fascinated some of the world's great intellects,

Gábor J. Székely is Professor, Department of Mathematics and Statistics, Bowling Green State University, Bowling Green, OH 43403-0221, and the Rényi Institute of the Hungarian Academy of Sciences, Budapest, Hungary. Donald St. P. Richards is Professor, Department of Statistics, Penn State University, University Park, PA 16802-2111 (E-mail: richards@stat.psu.edu). We are grateful to the editor, a referee, and Sándor Csörgô for comments, on an earlier version of this article, which led to a much improved revision. 2000 Mathematics Subject Classification: Primary 60F05; Secondary 60G50. for it was clear that standard mathematical approaches to this problem were not in harmony with commonsense reasoning.

In a letter to Bernoulli written on May 21, 1728, and mailed from London to Basel, Gabriel Cramer, originator of Cramer's rule for solving systems of linear equations, rephrased the problem "for simplicity" from one involving dice to coins. Then in 1738 Nikolaus' cousin, Daniel Bernoulli, presented to the Imperial Academy of Sciences in St. Petersburg an article that announced the paradox to the world. In his historically memorable article Daniel Bernoulli also proposed a solution to the paradox and, although the paradox was first announced to the world by Montmort (1713), the problem has come to be known as the St. Petersburg paradox.

This article reviews some of the history of attempts to resolve the St. Petersburg paradox and we recount some related problems, including modifications to the original paradox. We discuss in detail the fascinating article of Durand (1957) in which the St. Petersburg paradox is applied to the problem of estimating fair valuations for the shares of growth companies. Our conclusion is that recent extreme declines in stock prices was foretold by Durand's application of the St. Petersburg paradox.

\section{THE PARADOX}

Let $X$ denote a player's net profit in a game of chance taking place in a casino. Then the game is called fair if $\mathbb{E}(X)$, the expected value of $X$, is zero. Intuitively, a game is fair if, after a large number of independent repetitions, neither the player nor the casino has an advantage over each other.

As Feller (1945) observed in an extensive discussion, this definition of a fair game usually applies only if the variance of $X$ is finite. Otherwise, there will exist examples of fair games in which, with probability tending to one as the number of independent repetitions tends to infinity, the player will sustain a steadily increasing loss having order of magnitude smaller than the number of repetitions. Nevertheless, at least for the moment, we shall proceed according to this definition even if the variance of $X$ is not finite.

The St. Petersburg Paradox: Peter tosses a fair coin repeatedly until it shows heads. He agrees to pay Paul two ducats if it shows heads on the first toss, four ducats if the first head appears on the second toss, eight ducats if the first head appears on the third toss, sixteen if on the fourth toss, etc. How much should Peter charge Paul as an entrance fee to this game so that the game will be fair?

In this game, the potential payoff doubles after each toss; we refer to games of this type as St. Petersburg games. In Daniel Bernoulli's later statement of the paradox, the payoff for $k$ tosses is $2^{k-1}$ ducats, but we have chosen to work throughout with a payoff of $2^{k}$ ducats in order to simplify calculation of expectations.

To determine the amount Peter should charge Paul as an entrance fee so that the St. Petersburg game will be fair, we cal- 
culate Paul's expected payoff. Surprisingly, the game cannot be made fair, no matter how large the entrance fee.

To show this, we calculate Paul's expected payoff as follows. Let $k$ be any positive integer; then the probability that the game ends at the $k$ th toss is $2^{-k}$, at which time Peter will pay Paul $2^{k}$ ducats. Let $Y$ denote Peter's payout; then $Y$ is a random variable with possible values $2,4,8,16, \ldots$, and $P\left(Y=2^{k}\right)=2^{-k}, k=$ $1,2,3,4, \ldots$. Equivalently, $\log _{2} Y$ has a geometric distribution with probability of success $1 / 2$. Therefore the expected value of $Y$ is

$$
\mathbb{E}(Y)=\frac{1}{2} \cdot 2+\frac{1}{4} \cdot 4+\frac{1}{8} \cdot 8+\cdots=1+1+1+\cdots,
$$

proving that $Y$ has infinite expectation. This shows that no finite amount of money can be a fair entrance fee.

In short, Paul should be willing to pay an infinite price to enter this game. However, this is a requirement to which almost no rational person would agree or be able to satisfy. Although the calculation of Paul's expectation is mathematically correct, the paradoxical conclusion was regarded by many early probabilists as unacceptable.

Indeed, Nikolaus Bernoulli's motivation for formulating this problem stemmed from his study of games of chance. In its early days, the theory of games of chance concentrated heavily on notions of fairness, equity, and examples of fair games, and so Bernoulli was surprised and concerned by his example of a game in which the price of entrance appeared to be grossly unfair. As evidence of this unfairness, Paul is certain to win a finite amount, so it seems unfair to impose an infinite entrance fee. Moreover, it is highly probable that Paul will receive only a small payment, so that even a large, finite entrance fee seems unfair. For instance, the probability that Paul receives at most 32 ducats is $31 / 32$, or $96.875 \%$, and so an entrance fee of 10,000 ducats, say, seems unreasonable.

\section{SOME ATTEMPTS TO RESOLVE THE PARADOX}

Several probabilists suggested modifications to the St. Petersburg paradox in efforts to obtain acceptable solutions. For the most part, these modifications employed the concept of "utility" which may be defined for our purposes as follows (see DeGroot 1970, p. 90).

Consider a game in which a player can receive a reward from $\mathcal{R}$, a set of possible rewards. We assume a basis of preferences among rewards, so that a player can specify a complete ordering of $\mathcal{R}$. For simplicity, we suppose that $\mathcal{R}$ is a countable subset of the real line such that the player's ordering of rewards is the natural ordering on the real line. Regarding $\mathcal{R}$ as a probability space, the player's preference among rewards in $\mathcal{R}$ leads to preferences among the probability distributions on $\mathcal{R}$. If $P$ and $Q$ are probability densities on $\mathcal{R}$, then we write $P \prec Q$ if the player prefers $Q$ to $P$ and $P \preceq Q$ if the player does not prefer $P$ to $Q$.

For any probability density function $P$ on $\mathcal{R}$ and any realvalued function $g$ on $\mathcal{R}$, we use the notation

$$
\mathbb{E}_{P}(g):=\sum_{r \in \mathcal{R}} P(r) g(r)
$$

A real-valued function $U$ on $\mathcal{R}$ is called a utility function if, for any probability distributions $P$ and $Q$ on $\mathcal{R}$ such that both $\mathbb{E}_{P}(U)$ and $\mathbb{E}_{Q}(U)$ exist, $P \preceq Q$ if and only if $\mathbb{E}_{P}(U) \leq$ $\mathbb{E}_{Q}(U)$.

Nikolaus Bernoulli, in his letters to Montmort, proposed several solutions to the paradox. Bernoulli proposed the assignment of zero probability to large values of $k$ in (1), thereby truncating the series. In this context, Bernoulli is arguing in favor of a utility function $U$ for which $U(k)=0$ for sufficiently large $k$. Bernoulli's argument was that very large values of $k$ were "morally certain" not to occur so that, from a practical standpoint, one loses nothing by truncating (1). Still, Bernoulli did not find his own argument convincing, hence he sought the views of others.

Gabriel Cramer, the Comte de Buffon (well known for "Buffon's needle"), and others viewed the calculation of expectation in (1) as unacceptable in that it simply added the actual payoffs weighted by the corresponding probabilities. Cramer's basic idea was that the larger the size of a person's fortune, the smaller the "moral value" of a given increment in that fortune. Cramer proposed alternatives under which the value of a sum of money is measured through various utility functions, such as the square root, or the inverse of the amount, or by placing a limit on payouts, all of these devices being chosen so as to lead to a finite expectation. In adopting these methods, these authors also were proposing as a basic principle that additional sums of money have decreasing marginal utility.

For example let us suppose, like Buffon and Cramer, that the utility of money ceases to increase beyond a certain amount, say, one million ducats. Or equivalently, let us make the natural assumption that Peter has limited resources, in which case he necessarily must place a limit on the size of his payouts. Bearing in mind that $2^{19}<10^{6}<2^{20}$, it follows that $Y$, Peter's payout in ducats, is

$$
Y=\left\{\begin{array}{clc}
2^{k}, & \text { if } & 1 \leq k \leq 19 \\
10^{6}, & \text { if } \quad k \geq 20
\end{array}\right.
$$

Further, the corresponding probabilities are given by

$$
P(Y=y)=\left\{\begin{array}{cl}
1 / y, & y=2,4,8, \ldots, 2^{19}, \\
2^{-20}+2^{-21}+\cdots, & y=10^{6} .
\end{array}\right.
$$

The expected value of Peter's payouts is then seen to be

$$
\begin{aligned}
\frac{1}{2} \cdot 2+\frac{1}{4} \cdot 4 & +\cdots+\frac{1}{2^{19}} \cdot 2^{19} \\
& +\left(\frac{1}{2^{20}}+\frac{1}{2^{21}}+\cdots\right) 10^{6}=20.9073 \ldots \approx 21 .
\end{aligned}
$$

This proves that the game becomes slightly favorable to Peter if Paul is required to pay an entrance fee of 21 ducats.

Nikolaus Bernoulli was not satisfied with Cramer's solution, so he communicated the problem to his younger cousin Daniel Bernoulli who, at the time, was a member of the Imperial Academy of Sciences in St. Petersburg. Daniel responded in 1731 with a draft of his famous paper, published later in 1738 .

In that paper, Daniel Bernoulli concluded that the natural choice of utility function should be the logarithm function. Daniel argued that "in order to perceive the problem more correctly we shall assume that there is an imperceptibly small growth in the individual's wealth which proceeds continuously 
Table 1. Buffon's Simulation of the St. Petersburg Game

\begin{tabular}{ccc}
\hline \hline Tosses $(k)$ & Frequency & Payoff $\left(2^{k}\right)$ \\
\hline 1 & 1061 & 2 \\
2 & 494 & 4 \\
3 & 232 & 8 \\
4 & 137 & 16 \\
5 & 56 & 32 \\
6 & 29 & 64 \\
7 & 25 & 128 \\
8 & 8 & 256 \\
9 & 6 & 512 \\
\hline
\end{tabular}

by infinitesimal increments. Now it is highly probable that any increase in wealth, no matter how insignificant, will always result in an increase in utility which is inversely proportionate to the quantity of goods already possessed."

Thus, Daniel Bernoulli argued that if there is an infinitesimal increase, $\Delta x$, in wealth then $\Delta U$, the corresponding infinitesimal increase in utility should be inversely proportional to the size of the fortune already amassed. Then, for some constant $a$,

$$
\Delta U=(a / x) \Delta x .
$$

By perspicacious geometrical arguments, Bernoulli deduced that $U(x)=a \log x+b$, where $b$ is a constant. By contrast, modern methods would obtain from (2) the differential equation $d U / d x=a / x$, which may be integrated in a straightforward manner to return the solution given above.

For example, if the utility of an amount of money is measured by its logarithm then the expected utility arising from playing the St. Petersburg game is

$$
\mathbb{E}_{P}(\log Y)=\sum_{k=1}^{\infty} \frac{1}{2^{k}} \log \left(2^{k}\right)=\sum_{k=1}^{\infty} \frac{k}{2^{k}} \log 2=2 \log 2,
$$

where $P$ corresponds to the geometric distribution. This expectation corresponds to a monetary amount of exactly four ducats. From this point of view a rational gambler like Paul—that is, a person who measures marginal utility using logarithms - would pay any sum less than four ducats to enter the game.

Buffon had a child play the St. Petersburg game 2,048 times. The results of this simulation, reported by Jorland (1987, p. 168), are reproduced in Table 1.

Based on these empirical frequencies Buffon concluded that, despite the theoretical expected infinite expectation, the St. Petersburg game in practice becomes fair with an entrance fee of approximately ten ducats.

Whitworth (1901) proposed another resolution of the paradox which is independent of devices for measuring the utility of money. His solution considered the possibility of gambler's ruin, a natural concern for all gamblers with finite resources. Whitworth assumed that prudent gamblers would place at risk a fixed percentage, rather than a fixed amount, of their funds, and he developed a procedure for analyzing ventures that involve risk of ruin. It follows from Whitworth's study that Paul's entrance fee should depend on the size of his funds.

Feller (1945) proposed a different method to determine entrance fees which would make the St. Petersburg game fair. Suppose Paul chooses to play the game repeatedly. After $n$ games have been played, let $R_{n}$ denote the total entrance fees and let $S_{n}$ denote Paul's accumulated receipts. Let us call the game asymptotically fair if the ratio $S_{n} / R_{n}$ converges to 1 in probability as $n$ tends to infinity, that is, if for every $\epsilon>0$

$$
\lim _{n \rightarrow \infty} P\left(\left|\frac{S_{n}}{R_{n}}-1\right|<\epsilon\right) \rightarrow 1 .
$$

Feller proved that the St. Petersburg game becomes asymptotically fair if $R_{n}=n \log _{2} n$. From the calculation of Paul's expected payoff, we see also that the game cannot be asymptotically fair if there is a fixed and finite entrance fee per game, that is, if $R_{n}=c n$ where $c$ is a finite constant. However, if the entrance fee per game may depend on the number of games already played then, according to Feller's theorem, the St. Petersburg paradox is resolved; see Feller (1969, pp. 235-237).

Others have noted that the St. Petersburg game seems unrealistic, given that natural limits exist on the time and financial resources of Peter and Paul. From the point of view of those critics, the St. Petersburg problem is not even a paradox. As Durand (1957, p. 352) eloquently put it, "Peter and Paul are mortal; so, after a misspent youth, a dissipated middle age, and a dissolute dotage, one of them will die, and the game will cease-heads or no heads. Or again, Peter's solvency is open to question, for the stakes advance at an alarming rate ... Even if Peter and Paul agree to cease after 100 tosses, the stakes, though finite, stagger the imagination."

In an extensive assessment of the St. Petersburg game, Samuelson (1977) commented: “Of course Buffon's calculated ratio is nonsensical as a proposed estimate of the mathematical expectation of Paul per infinite Petersburg game. In 2, 048 tosses, Paul may win anything from 0 to $2^{2,048}$. Why take the result of 1 arbitrary sample? And how did Buffon score uncompleted games? If Buffon correctly applies 'the method of fair division' between Peter and Paul for games interrupted before their finish, he will find that half the time on the 2,048th toss the sequence shows a T and an incomplete game ... therefore, Paul's expected money gain will indeed be infinite ... Consequently, Buffon's Monte Carlo experiments are childish ways to avoid the true infinity in the Petersburg expectations: adultishly understood, they confirm the need to replace Paul's linear" utility function by a sufficiently concave function. "At bottom," Samuelson concluded, "the "paradox' is no paradox."

Following on the idea of varying entrance fees as initiated by Feller (1945), a deterministic sequence of entrance fees for the St. Petersburg game,

$$
242824216242824232242 \ldots \text {, }
$$

was given by Steinhaus (1949). To construct this sequence, place twos in alternating empty places, then fill every second empty place by a four, next fill every second remaining empty space by an eight, and so on. Denote by $a_{1}, a_{2}, \ldots$ the members of this sequence, and let $a_{n}$ be the entrance fee at the $n$th repetition of a St. Petersburg game. Steinhaus proved that the sample distribution function of $a_{1}, \ldots, a_{n}$ converges to the distribution function of $Y$ as $n \rightarrow \infty$.

More recently, Csörgő and Simons (1993) provided an extensive discussion of Steinhaus' sequence of entrance fees, and there now exists an extensive literature on asymptotic theory for 
St. Petersburg games. We refer to Adler (1990), Berkes, Csáki, and Csörgő (1999), Csörgő and Simons (1996), and Csörgô (2003) for recent developments in this area and for further references. Especially intriguing is the remarkable article by Csörgô and Simons (2002) which reveals new paradoxes within the structure of the classical St. Petersburg paradox; in particular, their results show that, paradoxically, there may be a very different outcome if $n$ distinct Pauls play one St. Petersburg game each than if one Paul plays $n$ games.

\section{RELATED PARADOXES AND GAMES}

Daniel Bernoulli's response to the paradox has not been universally regarded as satisfactory (see Weirich 1984), this being due to a controversy regarding the relationship between additional amounts of ducats and the measurement of utility. Moreover, even if it is assumed that additional sums of money have decreasing marginal utilities and also that it is appropriate to calculate utilities by taking logarithms, the problem is not entirely resolved. Indeed, Samuelson (1977) discussed at length the "Super-Petersburg" paradoxes constructed by K. Menger; here, for a given unbounded utility function, the payoffs grow sufficiently fast that the resulting expected utility is infinite. For instance, in a St. Petersburg game with logarithmic utility in which Paul receives $2^{2^{k}}$ ducats if the first head is obtained at the $k$ th toss, Paul's expected payoff again is infinite, and the paradox returns. If a new, unbounded utility function were to be devised for this game so as to return a finite expectation, then a new set of more rapidly increasing payoffs can be designed so that the resulting expectation again is infinite. By repeatedly adjusting the payoff strategy, it is seen that the measurement of utility by logarithms can always be made to result in an infinite expectation, and it becomes clear that the paradox can be avoided if and only if the utility function is bounded, an observation due to Menger.

Despite objections to the calculation of utilities by the use of logarithms, there are instances in which this approach is entirely natural and has significant advantages over other measures of utility. For one such example within the context of optimal gambling systems, we refer the reader to Thorp (1969, p. 289).

There are numerous paradoxes analogous to the St. Petersburg problem. One of them is the following paradox, communicated to Székely by Guttman in 1983: Peter entraps Paul into tossing a fair coin repeatedly. When $k$ tosses are needed to observe the first head, Paul receives $(-2)^{k}$ ducats from Peter. In other words, if $k$ is even then Paul receives $2^{k}$ ducats from Peter, but if $k$ is odd then Paul pays $2^{k}$ ducats to Peter. Should Paul be happy or unhappy about having been entrapped into playing this game? The answer is that Paul should both be happy and unhappy.

To explain this paradoxical conclusion, we partition the set of positive integers into subsets $\{1,2,4\},\{3,6,8\},\{5,10,12\}$, and so on, so that the $r$ th subset in this partition is $\{2 r-1,4 r-2,4 r\}$. Then the game can be viewed as consisting of a collection of subgames, each corresponding to one of the subsets in our partition. With probability $2^{-(2 r-1)}+2^{-(4 r-2)}+2^{-4 r}$, Paul has received the subgame which awards $(-2)^{k}$ ducats with probability

$$
\frac{2^{-k}}{2^{-(2 r-1)}+2^{-(4 r-2)}+2^{-4 r}}, \quad k=2 r-1,4 r-2,4 r .
$$

It is straightforward to calculate that each of these subgames is favorable to Paul, that is, Paul has positive expected payoff from each subgame. Therefore, Paul should be happy.

On the other hand, we can also partition the set of positive integers into subsets $\{1,2,3\},\{4,5,7\},\{5,10,12\}$, and so on, in which the $r$ th subset in this partition is $\{2 r, 4 r-3,4 r-1\}$. With probability $2^{-2 r}+2^{-(4 r-3)}+2^{-(4 r-1)}$, Paul receives the subgame which awards $(-2)^{k}$ ducats with probability

$$
\frac{2^{-k}}{2^{-2 r}+2^{-(4 r-3)}+2^{-(4 r-1)}}, \quad k=2 r, 4 r-3,4 r-1 .
$$

Each of these subgames is unfavorable to Paul, so he also should be unhappy.

Let us restate Guttman's problem in terms of conditional expectations. If $X$ and $Y$ are jointly distributed random variables, we denote by $P(d x \mid Y)$ the conditional probability distribution of $X$ given $Y$. Then the conditional expectation of $X$ given $Y$ is

$$
\int x P(d x \mid Y)
$$

Surprisingly, there exist random variables $X, Y$, and $Z$ such that $\mathbb{E}(X \mid Y)>0>\mathbb{E}(X \mid Z)$ with probability one. For example, let $X$ denote the overall reward to Paul in Guttman's game. Let $Y=r$ if Paul receives the $r$ th favorable game in which $X \in\{2 r-1,4 r-2,4 r\}$. Also, let $Z=r$ if Paul receives the $r$ th unfavorable game in which $X \in\{2 r, 4 r-3,4 r-1\}$. Then it is simple to verify that $\mathbb{E}(X \mid Y)>0>\mathbb{E}(X \mid Z)$ with probability one.

Stories of St. Petersburg games date to antiquity. There is the classic, perhaps apocryphal, story of the king who decided to reward an old and faithful peasant with a generous sum of gold. Instead, the peasant gently requested his king to give him one grain of corn on the first day of the month, two grains of corn on the second day, four grains on the third day, etc. The king was so pleased by his servant's deep modesty that he happily granted the request, and his kingdom was bankrupt before the month ended.

An interesting example of a St. Petersburg offer involved Ken Harrelson, a Major League baseball player with the Cleveland Indians from 1969-1971. As reported by Smith (1988, p. 157), Harrelson proposed to Gabe Paul, general manager of the Cleveland Indians, a St. Petersburg deal in which Harrelson would play the entire baseball season without salary "except for 50 cents doubled for every home run he hit." Harrelson's salary would then be 50 cents if he hit only one home run, one dollar if he hit two home runs, two dollars for three home runs, and so on. Gabe Paul was wise enough to decline Harrelson's offer, especially since Harrelson went on to hit 30 home runs during that season!

Currently, a popular television game show, Who Wants to be a Millionaire?, offers players the chance to win up to one million dollars. Players are offered a sequence of questions, each with four possible answers. If players answer a question correctly then they can walk away with their prize, or they can choose to answer a new question, thereby taking the risk of losing all if they answer incorrectly or doubling their prize by answering correctly. 
Many examples of St. Petersburg games and their generalizations are studied in the statistics, economics, and mathematics literature. A search of databases covering the literature in those fields will recover dozens of articles studying proposed solutions and applications.

\section{THE VALUATION OF GROWTH STOCKS}

A remarkable application of the St. Petersburg paradox, due to Durand (1957), relates to the valuation of the common stocks of "growth" companies. Here, a "growth" company is one whose revenues are growing significantly faster than the overall economy; and the stocks or shares of these companies, or even the companies themselves, are commonly referred to as "growth stocks." We shall review this application in detail because of its applicability to financial events in the late 1990s and early 2000s.

As background, we recall that by early 2000 , unprecedented increases had occurred over the previous three years in the prices of growth stocks. These increases in stock prices sparked a lively debate over whether investors were wise to purchase these shares, or foolish not to purchase greater amounts of shares before prices increased even further.

On the one hand were skeptics, including Alan Greenspan, chairman of the board of governors of the United States Federal Reserve System, who raised concerns about inflationary pressures resulting from increased stock prices. In a now-famous speech on December 5, 1996, Greenspan's question, "But how do we know when irrational exuberance has unduly escalated asset values, which then become subject to unexpected and prolonged contractions as they have in Japan over the past decade?," was followed by dramatic fluctuations on world financial markets.

On the other hand were believers, including many mutual funds which had purchased substantial numbers of shares of high-tech companies. Indeed, the Wall Street Journal reported on November 19, 1999, that 59 mutual funds had amassed increases of more than $100 \%$ during the period January 1-November 17, 1999. One fund, the Nicholas-Applegate Global Technology Fund, which specialized in high-tech stocks, had increased in price during the same period by the astounding amount of $325 \%$, or about $1 \%$ per day, an amount which the Journal commented, tongue-in-cheek, as possibly insufficient for "patient investors."

Following Durand (1957), we consider a modified St. Petersburg game in which "Peter" is a growth company and "Paul" is a prospective purchaser of Peter's stock. We assume that the probability of tossing heads is $i /(1+i), i>0$; then the probability of tails is $1 /(1+i)$.

Next, suppose that the corresponding payoffs are a series of increasing payments in which Peter pays Paul $D$ ducats if the first toss results in a tail, $D(1+g)$ ducats if the second toss is a tail, $D(1+g)^{2}$ ducats if the third toss is a tail, and so on, and this continues until the toss results in heads, at which point the game ends. If $k$ tosses are needed for the game to end then the total payment to Paul is

$$
\sum_{j=0}^{k-2} D(1+g)^{j}=\frac{D\left[(1+g)^{k-1}-1\right]}{g} .
$$

Because heads and tails appear with probabilities $i /(1+i)$ and $1 /(1+i)$, respectively, then the payment (3) occurs with probability $i /(1+i)^{k}$. As Durand (1957) observed, Paul's expected payoff is given by the double summation

$$
\sum_{k=1}^{\infty} \frac{i}{(1+i)^{k}} \sum_{j=0}^{k-2} D(1+g)^{j} .
$$

This double sum is evaluated by substituting for the inner sum the closed form expression (3). Alternatively, by reversing the order of summation and evaluating the resulting inner sum, we find that Paul's expected payoff is

$$
\sum_{k=1}^{\infty} \frac{D(1+g)^{k-1}}{(1+i)^{k}}=\left\{\begin{array}{clc}
D /(i-g), & \text { if } & g<i \\
\infty, & \text { if } & g \geq i
\end{array}\right.
$$

In summary, if $g<i$, then Paul's expected payoff is $D /(i-g)$. On the other hand, if $g \geq i$, then the infinite series diverges and Paul's expected payoff is infinite, in which case a realistic Paul may wisely decline to pay the corresponding entrance fee.

In the context of appraising the values of financial securities, the parameter $i$ represents a compound interest rate (or an effective rate of interest); equivalently, the present value of a loan of one dollar to be repaid one year in the future is $1 /(1+i)$. In the appraisal of fair value for a company's shares, $g$ represents the growth rate of the company as measured by the compound increase in revenue per share.

A widely accepted approach to estimating a fair value for Peter's stock is to discount all future dividends in perpetuity. Here, a fair value for Peter's stock is estimated by the present value of all future dividends. Denote by $E_{n}$ Peter's earnings (i.e., profits) per share in year $n$. Also, let $B_{n}$ denote Peter's book value, or net asset value, per share in the same year. Further, denote by $D_{n}$ the total of Peter's paid-out dividends per share in year $n$. A moment's reflection makes it clear that changes in book value from year-to-year are equal to the difference between earnings and dividends paid, that is, for all $n \geq 1$

$$
B_{n+1}-B_{n}=E_{n}-D_{n}
$$

In estimating fair value for Peter's stock, it is common practice for Paul to assume that the ratios $r=E_{n} / B_{n}$ and $p=D_{n} / E_{n}$ are independent of $n$. This assumption implies that $B_{n+1}-B_{n}$, the change in book value from year $n$ to year $n+1$, is a constant multiple of $E_{n}$ :

$$
B_{n+1}-B_{n}=E_{n}-D_{n}=(1-p) E_{n}=(1-p) r B_{n} .
$$

Therefore Peter's dividends, book value, and earnings all are growing at a constant rate, $g=(1-p) r$. In this context the sum (4) represents a perpetual series of dividend payments, starting at $D$ ducats, growing at a constant rate $g$, and discounted at rate $i$ in perpetuity. If $i>g$, then the sum (4) converges to $D_{1} /(i-g)=p E_{1} /(i-g)$, which represents an estimate of fair value for one share of Peter's stock.

If $i \leq g$, then the series (4) diverges, and we now have a form of the St. Petersburg paradox in which the practice of discounting future dividends at a uniform rate in perpetuity leads to a paradoxical result. 


\section{CONCLUDING REMARKS}

The St. Petersburg paradox explains some of the unprecedented increases in the prices of high-tech growth stocks in the late 1990s. During that period, the Federal Reserve System's discount rate was near a historical low; in the context of Durand's article, $i$ was very small. Moreover, purchasers of growth stocks assumed that $g$, the growth rate of a typical high-tech company, would remain high in perpetuity. The outcome was that $i<g$; indeed, even more extreme was that for many hightech companies, $i / g \simeq 0$. By discounting earnings and dividends in perpetuity, any use of the valuation formula (4) led to estimated valuations of tech-stock share prices at levels as high as the appraiser cared to make them.

Having applied the valuation formula (4) to obtain exorbitant estimated valuations for many high-tech growth stocks, stock purchasers bought avidly, thereby forcing prices to extreme levels. By late 2000, stock prices underwent the "prolonged contractions" predicted by Greenspan, with subsequent unprecedented losses to corporate and individual stock buyers. Three years later, many formerly avidly sought-after high-tech companies and mutual funds were defunct.

Purchasers of high-tech growth stocks in the late 1990s overlooked completely the cautionary analyses of Durand (1957) and Malkiel (1963) regarding the perpetual discounting of earnings or dividends; even more regrettable was the propensity to ignore the sage advice of Graham (1985). It is striking that Durand's article was completely overlooked in the 1990s despite the fact that it was written during a similar period in the 1950s when stock purchasers had been eagerly purchasing the high-tech stocks of the time, and despite the clear observation by Durand and earlier authors that the divergence of the series (4) when $i<g$ would lead to infinite estimated fair prices for common stocks. As a clear warning, Durand (1957, p. 349), quoting Clendenin and Van Cleave (1954), commented that he had "not yet seen any growth stocks marketed at the price of infinity dollars per share, but [would] hereafter be watching." Unfortunately, these comments were overlooked or were unpersuasive to high-tech stock buyers of the 1950s and they eventually came to grief, as did similar buyers in the 1990s.

We close with two comments from Mark Twain who noted, for the benefit of stock market speculators, that

There are two times in a man's life when he should not speculate: when he can't afford it, and when he can.

Also, from Twain's Pudd'nhead Wilson's Calendar comes the quotation,

October. This is one of the peculiarly dangerous months to speculate in stocks in The others are July, January, September, April, November, May, March, June, December, August and February.

[Received October 2003. Revised March 2004.]

\section{REFERENCES}

Adler, A. (1990, "Generalized One-Sided Laws of the Iterated Logarithm for Random Variables Barely With or Without Finite Mean," Journal of Theoretical Probability, 3, 587-597.
Berkes, I., Csáki, E., and Csörgő, S. (1999), "Almost Sure Limit Theorems for the St. Petersburg Game," Statistics \& Probability Letters, 45, 23-30.

Bernoulli, D. (1738), "Specimen Theoriae Novae de Mensura Sortis," Commentarii Academiae Scientiarum Imperialis Petropolitanea, V, 175-192. (Translated and republished as "Exposition of a New Theory on the Measurement of Risk," (1954), Econometrica, 22, 23-36.)

Bru, B., Bru, M.-F., and Chung, K.-L. (1999), "Borel et la Martingale de SaintPetersbourg," Revue d'Histoire des Mathématiques, 5, 181-247.

Clendenin, J. C., and Van Cleave, M. (1954), "Growth and Common Stock Values," The Journal of Finance, 9, 365-376.

Csörgô, S. (2003), "Merge Rates for Sums of Large Winnings in Generalized St. Petersburg Games," Acta Scientiarum Mathematicarum (Szeged), 69, 441454.

Csörgô, S., and Simons, G. (1993-1994), "On Steinhaus' Resolution of the St. Petersburg Paradox," Probability and Mathematical Statistics, 14, 157-172. (1996), "A Strong Law of Large Numbers for Trimmed Sums, with Applications to Generalized St. Petersburg Games," Statistics \& Probability Letters, 26, 65-73.

(2002), "The Two-Paul Paradox and the Comparison of Infinite Expectations," in Limit Theorems in Probability and Statistics, eds. I. Berkes et al., Budapest: János Bolyai Mathematical Society, pp. 427-455.

DeGroot, M. H. (1970), Optimal Statistical Decisions, New York: McGraw-Hill.

Dehling, H. G. (1997), "Daniel Bernoulli and the St. Petersburg Paradox," Nieuw Archief voor Wiskunde, 15, 223-227.

Durand, D. (1957), "Growth Stocks and the Petersburg Paradox," The Journal of Finance, 12, 348-363.

Dutka, J. (1988), "On the St. Petersburg Paradox," Archive for History of Exact Sciences, 39, 13-39.

Feller, W. (1945), "Note on the Law of Large Numbers and 'Fair' Games," Annals of Mathematical Statistics, 16, 301-304.

Graham, B. (1985), The Intelligent Investor: A Book of Practical Counsel (4th ed.), New York: Harper \& Row.

Jorland, G. (1987), "The Saint Petersburg Paradox 1713-1937," in The Probabilistic Revolution (vol. 1), eds. L. Krüeger, et al., Cambridge, MA: MIT Press, pp. 157-190.

Malkiel, B. (1963), "Equity Yields, Growth, and the Structure of Share Prices," American Economic Review, 53, 1004-1031.

de Montmort, P. R. (1713), Essay d'Analyse sur les Jeux de Hazard (2nd ed.) Paris.

Samuelson, P. A. (1977), "St. Petersburg Paradoxes: Defanged, Dissected, and Historically Described," Journal of Economic Literature, 15, 24-55.

Smith, G. (1988), Statistical Reasoning (2nd ed.), Boston, MA: Allyn \& Bacon.

Steinhaus, H. (1949), "The So-Called Petersburg Paradox," Colloquium Mathematicum, 2, 56-58.

Thorp, E. O. (1969), "Optimal Gambling Systems for Favorable Games," Reviews of the International Statistical Institute, 37, 273-293.

Weirich, P. (1984), "The St. Petersburg Gamble and Risk," Theory and Decision, 17, 193-202.

Whitworth, W. A. (1901), Choice and Chance (5th ed.), Cambridge: Deighton, Bell, \& Co.

\section{ADDITIONAL READING}

The following references provide additional information on asymptotic approaches to resolving the paradox and the method of variable entrance fees.

Csörgő, S. (2002), "Rates of Merge in Generalized St. Petersburg Games," Acta Scientiarum Mathematicarum (Szeged), 68, 815-847.

Csörgő, S., and Dodunekova, R. (1991), "Limit Theorems for the Petersburg Game," in Sums, Trimmed Sums and Extremes, eds. M. G. Hahn et al., Boston: Birkhäuser, pp. 285-315.

Feller, W. (1969), An Introduction to Probability Theory and Its Applications (vol. I, 2nd ed.), New York: Wiley.

Martin-Löf, A. (1985), "A Limit Theorem Which Clarifies the 'Petersburg Paradox'," Journal of Applied Probability, 22, 634-643. 
The following references provide additional information on the St. Petersburg paradox and its connections with the concept of marginal utility.

Arntzenius, F., and McCarthy, D. (1997), "The Two Envelope Paradox and Infinite Expectations," Analysis, 57, 42-50.

Machina, M. J. (1982), “'Expected Utility’ Analysis Without the Independence Axiom," Econometrica, 50, 277-323.

Shafer, G. (1988), “The St. Petersburg Paradox,” in Encyclopedia of Statistical Sciences, Volume 8, eds. N. L. Johnson and S. Kotz, New York: Wiley, pp. 865-870.
Sinn, H.-W. (1989), Economic Decisions Under Uncertainty (2nd ed.), Heidelberg: Physica-Verlag.

The following reference provides additional information on the St. Petersburg paradox and its application to the valuation of growth stocks.

Bernstein, P. L. (1996), Against the Gods: The Remarkable Story of Risk, New York: Wiley. 